\title{
Effect of mirror curvature on the angular resolution of silicon pore optics
}

Jegers, Arne S.; Ferreira, Desiree Della Monica; Bergbäck Knudsen, Erik; Massahi, Sonny; Svendsen, Sara; Lindquist Henriksen, Peter; Gellert, Nis Christian; Sironi, Giorgia; Spiga, Daniele; Ferreira, Ivo Total number of authors:

12

\section{Published in:}

Proceedings of SPIE

Link to article, DOI:

$10.1117 / 12.2594530$

Publication date:

2021

Document Version

Publisher's PDF, also known as Version of record

Link back to DTU Orbit

Citation (APA):

Jegers, A. S., Ferreira, D. D. M., Bergbäck Knudsen, E., Massahi, S., Svendsen, S., Lindquist Henriksen, P., Gellert, N. C., Sironi, G., Spiga, D., Ferreira, I., Shortt, B., \& Bavdaz, M. (2021). Effect of mirror curvature on the angular resolution of silicon pore optics. In S. L. O'Dell, J. A. Gaskin, \& G. Pareschi (Eds.), Proceedings of SPIE: Optics for EUV, X-Ray, and Gamma-Ray Astronomy X [118220F] SPIE - International Society for Optical Engineering. Proceedings of SPIE - The International Society for Optical Engineering Vol. 11822 https://doi.org/10.1117/12.2594530

\section{General rights}

Copyright and moral rights for the publications made accessible in the public portal are retained by the authors and/or other copyright owners and it is a condition of accessing publications that users recognise and abide by the legal requirements associated with these rights.

- Users may download and print one copy of any publication from the public portal for the purpose of private study or research.

- You may not further distribute the material or use it for any profit-making activity or commercial gain

- You may freely distribute the URL identifying the publication in the public portal 


\title{
Effect of mirror curvature on the angular resolution of Silicon Pore Optics
}

\author{
Arne S Jegers $^{\mathrm{a}}$, Desiree Della Monica Ferreira ${ }^{\mathrm{a}}$, Erik Bergbäck Knudsen ${ }^{\mathrm{a}}$, Sonny Massahi ${ }^{\mathrm{a}}$, \\ Sara Svendsen ${ }^{\mathrm{a}}$, Peter Lindquist Henriksen ${ }^{\mathrm{a}}$, Nis Christian Gellert ${ }^{\mathrm{a}}$, Giorgia Sironi ${ }^{\mathrm{b}}$, Daniele \\ Spiga $^{b}$, Ivo Ferreira ${ }^{c}$, Brian Shortt ${ }^{c}$, and Marcos Bavdaz ${ }^{c}$ \\ aTechnical University of Denmark, DTU Space, Kongens Lyngby, Denmark \\ bINAF - IASF Milano, Milano, Italy \\ ${ }^{\mathrm{c}}$ European Space Agency, Noordwijk, The Netherlands
}

\begin{abstract}
Silicon Pore Optics are a novel technology for focusing high-energy photons, where pairs of mirror plates stacked into mirror modules. This paper presents a study of the angular resolution of SPO mirror assemblies, using a selection of sagittal mirror curvatures. Results were achieved by ray-tracing the different configurations of the mirror with the ray tracing software SPORT, or Silicon Pore Optics Ray Tracer, using an observation of the Crab Nebula originally obtained by Chandra.

It is found that a true Wolter geometry most closely reproduces the original image, while geometries with a conic approximation on either their primary or secondary mirrors create the most diffuse image. A geometry with conic approximations on both primary and secondary mirrors falls in between the two extremes.
\end{abstract}

\section{INTRODUCTION}

For ESA's upcoming Athena mission, a new type of Wolter I optics was developed. They use a new technology, dubbed Silicon Pore Optics, or SPO, with which mirrors consist of a number of coaligned 'mirror modules'. Each mirror module is itself composed of 4 stacks of confocal mirror plates, curved both sagitally and azimuthally to focus incoming light. Within a stack, mirrors are bonded together through radial ribs, giving rise to the channels, or 'pores', that the technology is named for. ${ }^{1}$ ?

This study presents the difference in the angular resolutions of SPO mirror assemblies with various sagittal curvatures. It will investigate the theoretical ideal curvature, where each mirror coincides with the paraboloid or hyperboloid surfaces appropriate for its position in the optics. ${ }^{2}$ Along with this configuration, various forms of the conic approximation of the paraboloid and/or the hyperboloid mirrors will be applied. ${ }^{3}$

\subsection{SPORT and McXtrace}

The software used to generate the ray-traces for this study is SPORT, the Silicon Pore Optics Ray-Tracer; it is a software tool developed as part of the SIMPOSIUM project. ${ }^{4}$ While it was designed with the Athena mission in mind, it is intended and capable as a general-purpose ray-tracer for Wolter I optics. It builds on McXtrace, ${ }^{5}$ the Monte Carlo X-ray tracer, and uses its AstroX package ${ }^{6}$ to simulate the inner workings of the SPO mirror modules.

\section{DATA ACQUISITION}

The data used in this paper is produced as follows: First, a model of an SPO mirror assembly is constructed in SPORT. Multiple variations of this model are then created, each with different sagittal curvatures for their primary and/or secondary mirrors. These optics variations are then ray-traced, first with star-like point sources at multiple incidence angles, then with an extended source on-axis.

\footnotetext{
Optics for EUV, X-Ray, and Gamma-Ray Astronomy X, edited by Stephen L. O'Dell,

Jessica A. Gaskin, Giovanni Pareschi, Proc. of SPIE Vol. 11822, 118220F · C

2021 SPIE · CCC code: 0277-786X/21/\$21 - doi: 10.1117/12.2594530

Proc. of SPIE Vol. 11822 118220F-1
} 
The mirror design used in this study can be seen in figure 1 is based loosely on that of the Athena mission - though it is not representative of Athena's performance. It has 600 mirror modules in its assembly, arranged in rings. Mirror modules are placed with their centers along a spherical shell centered on the focal point, in accordance with a Wolter-Schwarzschild mirror geometry. ${ }^{7}$

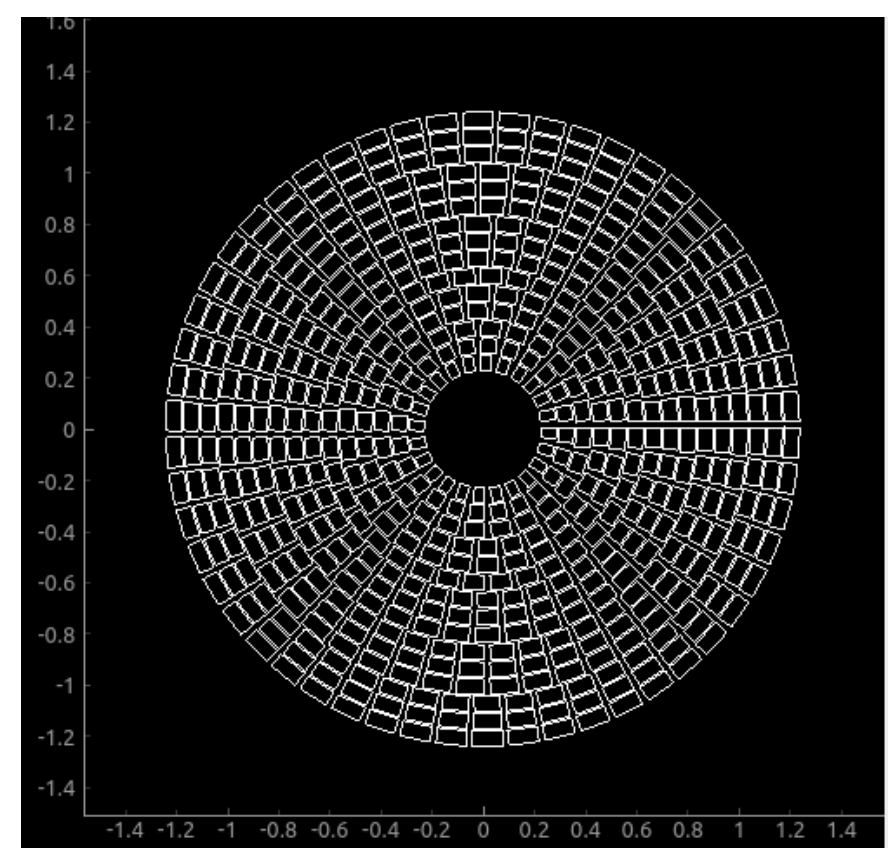

Figure 1. Schematic of the mirror design used in the study, as seen from the front. Each rectangle represents a single mirror module. Axes are both in meters.

An $\mathrm{Ir} / \mathrm{B}_{4} \mathrm{C}$ coating is used, with surface roughness of $0.45 \mathrm{~nm}$ is assumed, and is accounted for by using a reflectivity table as a function of photon energy and grazing angle. No diffuse scattering is included, i.e. in terms of geometry, the mirror surface is assumed to be perfectly smooth.

\subsection{Optics and source configurations}

Four mirror curvatures will be assessed: The first is the true Wolter curvature, where the mirrors follow their appropriate theoretical paraboloid and hyperboloid surfaces. The second and third configurations have, respectively, their primary and secondary mirrors shaped as conic approximations of the theoretical surfaces. Finally, the fourth configuration uses conic approximations for both primary and secondary stacks. These will be referred to as TW, PC, $\mathrm{CH}$ and $\mathrm{CC}$ in figures, respectively.

Using these optics configurations, two sets of ray-traces have been produced. The first was made using an infinitely distant point source, modeled by flooding the optics with parallel rays. For each mirror assembly, the source was placed on-axis, and at inclinations of 1', 10' and 20'. Its energy was kept constant at $1 \mathrm{keV}$.

The second set was made by ray-tracing an extended source on-axis. The source in question is the Crab nebula, and in particular, an observation from the Chandra X-ray observatory was used. This image was chosen for its high angular resolution, which better allows testing the limits of each design.

\section{POINT SOURCE PERFORMANCE}

Figures 2 and 3 show the focal plane responses of some of the point source ray-traces. Given this source geometry, these responses can be interpreted as point-spread functions of their respective optics, at their respective incidence angles. Note that pixel sizes may vary between individual FPR's. 

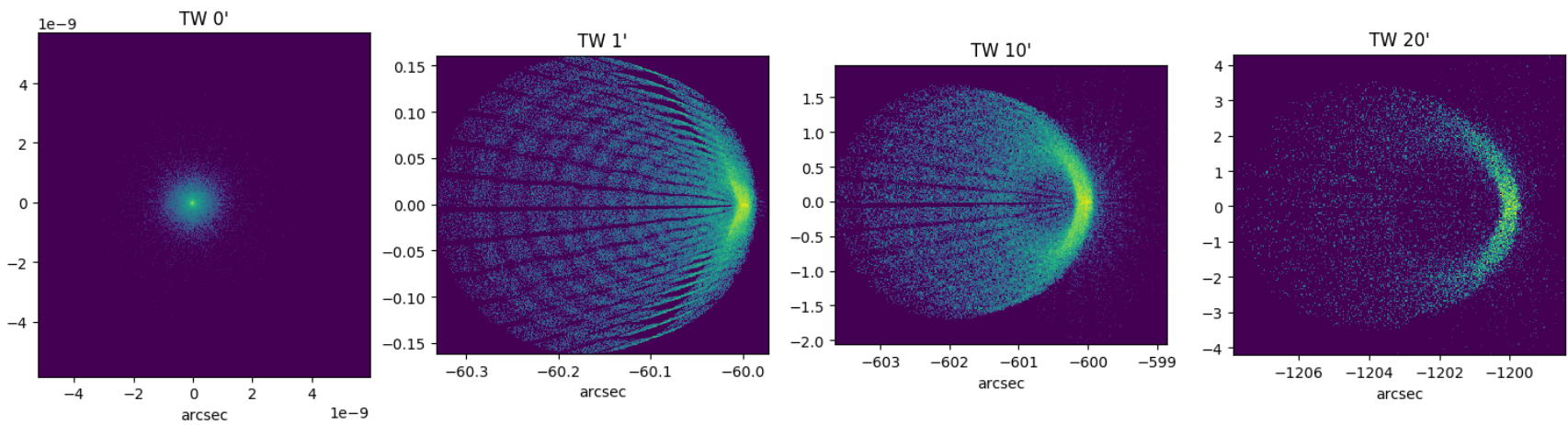

Figure 2. Point sources ray-traced at several off-axis angles, through true Wolter optics. The color maps are logarithmic, and each flux map is normalized to its own maximum.
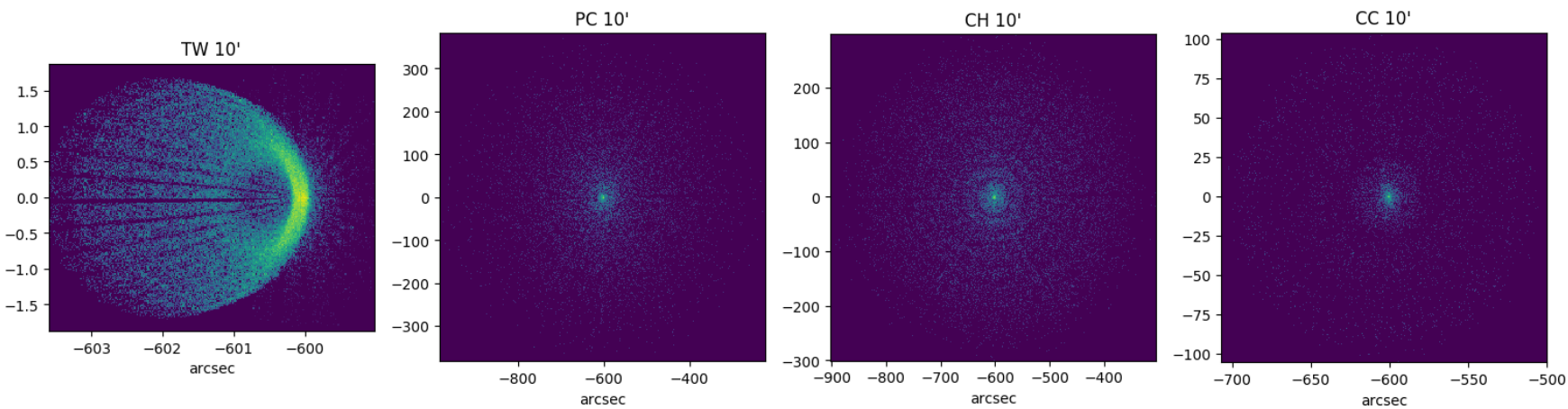

Figure 3. Point sources ray-traced at 10' off-axis, through the different optics configurations. The color maps are logarithmic, and each flux map is normalized to its own maximum.

Figure 2 shows the PSF of the TW geometry, at the different inclinations, while figure 3 shows the PSF at 10' for all geometries. The shape of the PSF is seen to be complex, and varies between inclinations and geometries. For the true Wolter geometry, it consists of a asymmetric circular pattern, while for the conic approximation geometries it consists of a very narrow peak, surrounded by a large halo.

Table 1 shows the radii that encircle $50 \%$ and $90 \%$ of the focal plane responses, as measured from their centroids.

The true Wolter geometry produces the sharpest PSF's, regardless of incidence angle. Note especially the on-axis PSF, with $90 \%$ of its intensity within just over a nanoarcsecond from the centroid. Also of note are the single conic approximation geometries, which perform noticeably worse than the double conic approximation. Their sizes also seem to change very little across different inclinations.

\section{EXTENDED SOURCE PERFORMANCE}

Figure 4 shows the focal plane responses that result from ray-tracing the crab nebula through each of the four mirror configurations. The flux maps have been generated with the same resolution as that of the original image, around 0.21 " per pixel in both directions. 
$50 \%$ and $90 \%$ intensity encircled radii

\begin{tabular}{|c|c|c|c|}
\hline Curvature & Inclination & $50 \%$ radius & $90 \%$ radius \\
\hline & $0^{\prime}$ & $3.89 \cdot 10^{-10} "$ & $1.09 \cdot 10^{-9} "$ \\
\hline \multirow[t]{3}{*}{ TW } & 1 ' & $8.46 \cdot 10^{-2} "$ & $0.16 "$ \\
\hline & $10 '$ & $0.92 "$ & $1.70 "$ \\
\hline & 20 ' & $1.98 "$ & $3.63 "$ \\
\hline & $0^{\prime}$ & $120.15 "$ & $252.77^{\prime \prime}$ \\
\hline \multirow[t]{3}{*}{$\mathrm{PC}$} & $1 '$ & 120.02" & $252.87 "$ \\
\hline & $10^{\prime}$ & $120.04 "$ & $257.54 "$ \\
\hline & 20 ' & 121.22" & 265.90" \\
\hline & $0^{\prime}$ & 116.26" & $218.50 "$ \\
\hline \multirow[t]{3}{*}{$\mathrm{CH}$} & 1 & 116.59" & 218.44" \\
\hline & $10^{\prime}$ & $119.17 "$ & $220.69 "$ \\
\hline & $20^{\prime}$ & 120.52" & $222.78 "$ \\
\hline \multirow{4}{*}{$\mathrm{CC}$} & $0^{\prime}$ & $28.13^{\prime \prime}$ & $85.68^{\prime \prime}$ \\
\hline & $1 '$ & $28.07 "$ & $85.55 "$ \\
\hline & $10^{\prime}$ & $37.67 "$ & $86.42 "$ \\
\hline & $20^{\prime}$ & $44.08 "$ & $87.54 "$ \\
\hline
\end{tabular}

Table $1.50 \%$ and $90 \%$ intensity encircled radii for each of the combinations of source angle and optics configuration.

As the point source study predicted, the true Wolter ray-trace is the closest to the original, while the conic approximations all produce less sharp images.

To quantify the sharpness of the images generated by the ray-traces, a 2D Fourier transform is generated from each. This produces a 2D power spectrum of said images, which indicates how much of the information in the images is encompassed by particular spatial frequencies. Figure 5 shows the $2 \mathrm{D}$ spectra taken from the images in figure 4 . They have been oriented such that the center pixel represents frequency $(0,0)$, i.e. a constant value across the entire image.

All spectra show the same overall pattern of a strong central peak, which quickly falls off away from the center. The spectra of the sharpest images, the original and the true Wolter, show a nearly vertical streak through the center. It is caused by a dark band in the original image due to a detector gap, which only the true Wolter image is sharp enough to recreate.

To compare these 2D spectra to one another, they are collapsed to 1 dimension by radially integrating around the $(0,0)$ frequency. Figure 6 shows these integrated power spectra, plotted by half wavelength as opposed to frequency. The half wavelength is chosen, as this corresponds to the FWHM of the sinusoid curves that the spectrum describes. Note that frequency $(0,0)$ is not included, as its corresponding wavelength would be infinite.

It can be seen that each curve starts at some noise level for its smallest features. When it reaches a threshold feature size, the amplitude of the spectrum rises, and the curves eventually converge. The threshold value where the curve rises from the noise gives an indication of the smallest features that any given configuration of optics would be able to resolve. Features smaller than the threshold size would be blurred beyond recognition. To find this threshold value, a piece-wise function given in equation 1 is fitted to the spectrum.

$$
f(x)= \begin{cases}b+c & x<x_{t} \\ \sqrt{a-\frac{a\left(x-x_{t}\right)}{b}} & x>=x_{t}\end{cases}
$$

This function is fitted to the logarithms of both $\mathrm{x}$ - and $\mathrm{y}$ - values, and consists of a constant value below the threshold size $x_{t}$, and the positive half of a hyperbola above it. The values for $x_{t}$ found by fitting this function 

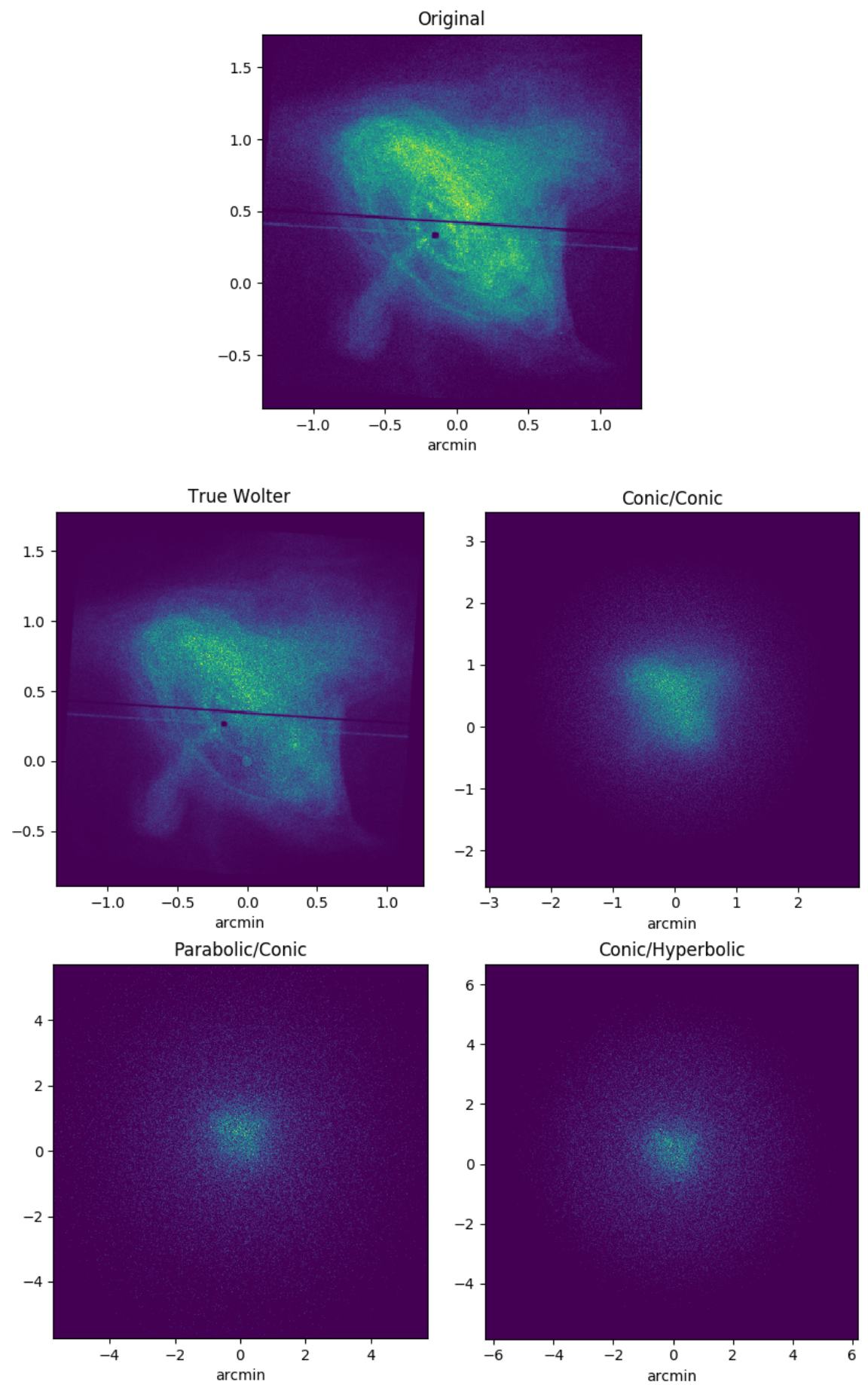

Figure 4. Focal plane responses from ray-traces of the crab nebula through the 4 different mirror configurations, normalized to their respective maximum values. Absolute pixel values are arbitrary. The original image is included at the top. Note the diagonal band across the original and TW ray-trace, likely due to a gap between detectors on the source image.

to each spectrum are shown in table $2 .^{*}$

${ }^{*}$ The actual amplitudes of the spectra are of less importance, and should not be compared since the images are normalized independently. 

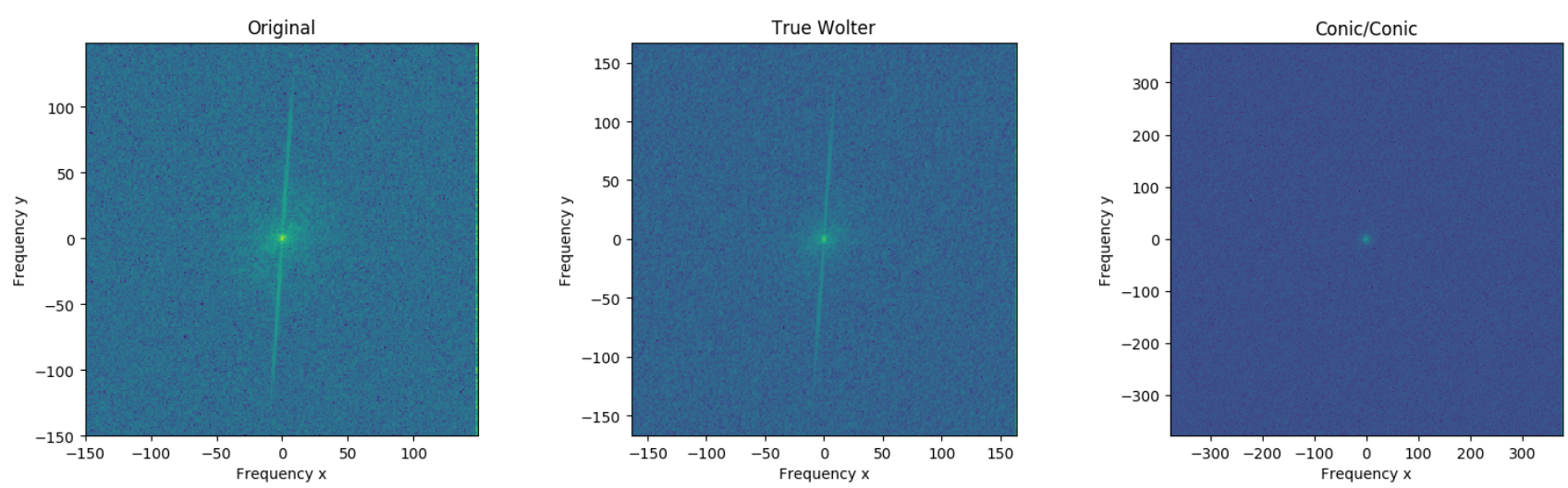

Figure 5. 2D Fourier transforms of a selection of the flux maps in figure 4, with frequency $(0,0)$ at the center of each graph (spectra for PC and $\mathrm{CH}$ are omitted as they are not visually distinct from that of CC). Each spectrum has a strong central peak, and tapers off toward higher frequencies. Note the strong, nearly vertical line across the spectra of both the original image and the true Wolter ray-trace. This is caused by the detector gap visible in the source image.

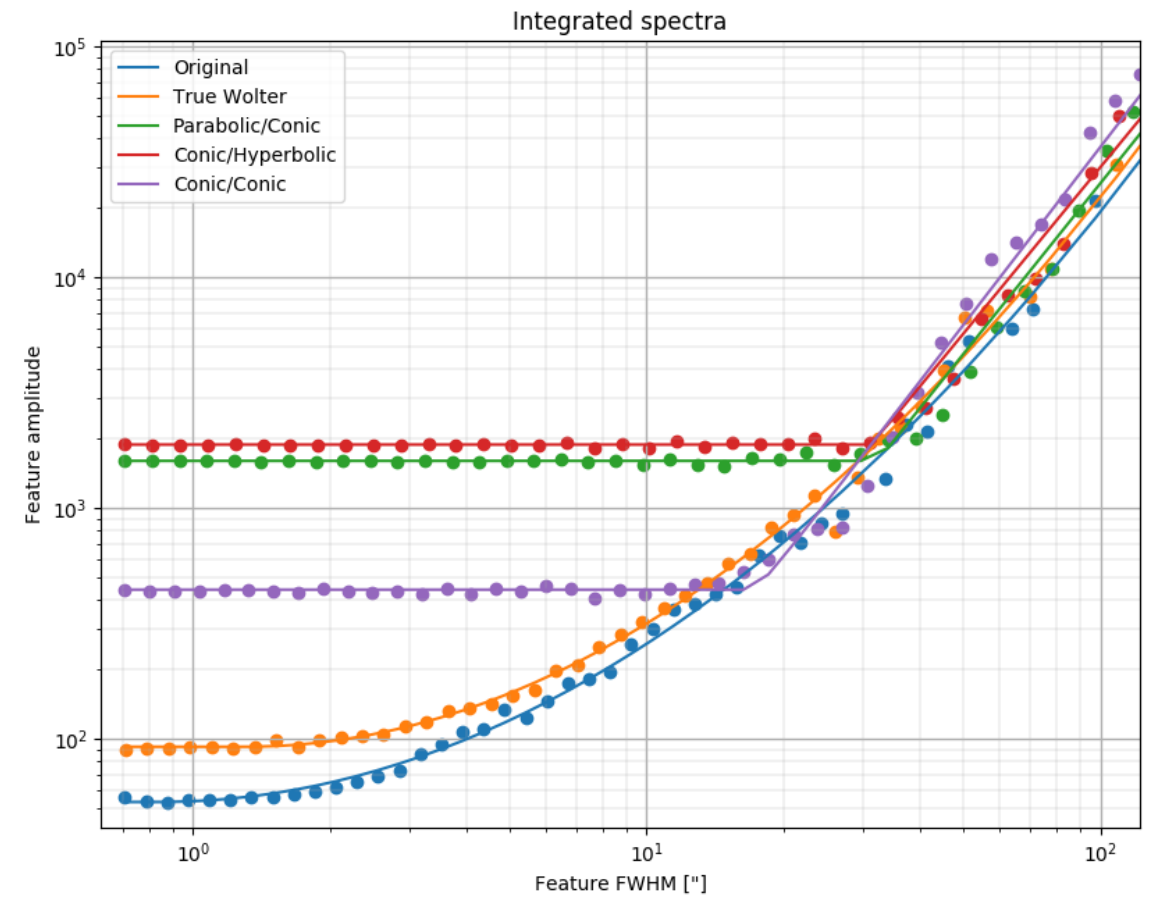

Figure 6. Radially integrated Fourier transforms of each of the flux maps in figure 4, plotted by half-wavelength to correspond to a feature FWHM. Overplotted are fitted piece-wise hyperbolas, as described in equation 1

The same trend as in the point source analysis appears here: the original image is the sharpest, closely followed by the true Wolter ray-trace. The single conic approximation ray-traces perform the worst, while the double conic approximation falls in between. The difference between single and double conic approximation is, however, slightly less severe than their respective PSF's would indicate.

\section{DISCUSSION}

While the results clearly point out the single conic approximation geometries as the worst options, it should be noted that their designs have not been optimized for these mirror curvatures. It is possible that, through adjusting the angles and positions of the mirror plates, better results can be achieved. That said, the results 


\begin{tabular}{lr}
\multicolumn{2}{c}{ Threshold feature sizes } \\
\hline Curvature & Threshold size \\
\hline Original & $0.84 "$ \\
TW & $1.31 "$ \\
PC & $32.29 "$ \\
CH & $31.39 "$ \\
CC & $16.71 "$
\end{tabular}

Table 2. Minimum feature sizes that can be distinguished from noise, as found by fitting the piece-wise hyperbola function defined in equation 1 . The values follow the same trend as in table 1.

show that a simple substitution of one of the mirror stacks with a conic approximation is not sufficient.

It is perhaps surprising that the difference between the minimum feature sizes of the original image and the true Wolter is as large as it is, given the minute size of the true Wolter PSF, even at 1' off-axis, roughly the extent of the source. A suspected contributing factor could be noise in the ray-trace, which is slightly higher than that in the original image (standard deviations across the entire images of 1.33 and 1.17, respectively).

It is also important to note that the reference design for Athena's mirror specifies a polynomial approximation for the sagittal curvature of its mirrors, which is not presented in this paper. Indeed, in Collon et. al (2019) it is found that the mirrors approach, and in some cases surpass, the 5 arcsecond resolution condition set for the mission. ${ }^{8}$ This is significantly better than the conic approximation designs presented in this study, only exceeded by the theoretical ideal of the true Wolter.

For this reason, and as stated before, the angular resolution figures observed in this paper do not reflect those of Athena's reference design. A further study could include this curvature for an assessment of Athena itself.

\section{CONCLUSION}

This study shows the impact of different mirror curvatures on the performance of a hypothetical SPO mirror assembly, in terms of angular resolution. It tests this using ray-traces of point sources, and of a Chandra observation of the crab nebula.

Of the variants examined in this study, the true Wolter performs the best, with a ray-trace very close to the original source image. The single conic approximation optics perform worst, while the double conic approximation falls in between. The poor performance of the single approximation variants is attributed to the geometry as a whole requiring optimization toward those curvatures to work properly.

The angular resolutions presented do not currently represent the reference design for Athena, which performs close to the true Wolter. Future studies could consist of including the polynomial approximation as a curvature in order to assess Athena directly, and investigating the impact of noise on the observed angular resolution of an image. Evaluating the effect at different energies presents another possible study.

\section{REFERENCES}

[1] D. Barret, A. Decourchelle, A. Fabian, M. Guainazzi, K. Nandra, R. Smith, and J. den Herder, "The athena space x-ray observatory and the astrophysics of hot plasma $\dagger, "$ Astronomische Nachrichten, vol. 341, p. 224-235, Feb 2020.

[2] L. P. VanSpeybroeck and R. C. Chase, "Design parameters of paraboloid-hyperboloid telescopes for x-ray astronomy," Appl. Opt., vol. 11, pp. 440-445, Feb 1972.

[3] R. Willingale, G. Pareschi, F. Christensen, and J.-W. den Herder, "The hot and energetic universe: The optical design of the athena+ mirror," 2013.

[4] G. Sironi, D. Spiga, D. D. M. Ferreira, I. Ferreira, M. Bavdaz, E. B. Knudsen, G. Bianucci, F. E. Christensen, M. Collon, F. Marioni, G. Pareschi, A. S. Jagers, B. Salmaso, B. Shortt, G. Tagliaferri, G. Vacanti, P. Conconi, G. Valsecchi, N. J. Westergaard, E. Wille, and S. Massahi, "Simulating the optical performances of the 
ATHENA x-ray telescope optics," in Space Telescopes and Instrumentation 2018: Ultraviolet to Gamma Ray (J.-W. A. den Herder, S. Nikzad, and K. Nakazawa, eds.), vol. 10699, pp. 933 - 942, International Society for Optics and Photonics, SPIE, 2018.

[5] E. B. Knudsen, A. Prodi, P. Willendrup, K. Lefmann, J. Baltser, C. Gundlach, M. S. del Rio, C. Ferrero, and R. Feidenhans'l, "McXtrace: a modern ray-tracing package for x-ray instrumentation," in Advances in Computational Methods for X-Ray Optics II (M. S. del Rio and O. Chubar, eds.), vol. 8141, pp. 130 - 136, International Society for Optics and Photonics, SPIE, 2011.

[6] E. B. Knudsen, D. D. M. Ferreira, N. J. Westergaard, F. E. Christensen, S. Massahi, B. Shortt, I. Ferreira, and D. Spiga, "The McXtrace AstroX toolbox: a general ray tracing software package for end to end simulation of x-ray optics for astronomical instrumentation," in Space Telescopes and Instrumentation 2018: Ultraviolet to Gamma Ray (J.-W. A. den Herder, S. Nikzad, and K. Nakazawa, eds.), vol. 10699, pp. 952 - 958, International Society for Optics and Photonics, SPIE, 2018.

[7] R. C. Chase and L. P. VanSpeybroeck, "Wolter-schwarzschild telescopes for x-ray astronomy," Appl. Opt., vol. 12, pp. 1042-1044, May 1973.

[8] M. J. Collon, G. Vacanti, N. M. Barrière, B. Landgraf, R. Günther, M. Vervest, L. Voruz, S. Verhoeckx, L. Babić, L. Keek, D. Girou, B. Okma, E. Hauser, M. W. Beijersbergen, M. Bavdaz, E. Wille, S. Fransen, B. Shortt, I. Ferreira, J. Haneveld, A. Koelewijn, R. Start, M. Wijnperle, J. . Lankwarden, C. Van Baren, P. Hieltjes, J. W. Den Herder, P. Müller, E. Handick, M. Krumrey, M. Bradshaw, V. Burwitz, G. Pareschi, S. Massahi, S. Svendsen, D. Della Monica Ferreira, F. E. Christensen, G. Valsecchi, P. Oliver, I. Chequer, and K. Ball, "Status of the silicon pore optics technology," in Proceedings of SPIE - The International Society for Optical Engineering, vol. 11119, 2019. Cited By :10. 